\title{
HOMOCLINIC ORBITS \\ OF FIRST ORDER NONLINEAR HAMILTONIAN SYSTEMS WITH ASYMPTOTICALLY LINEAR NONLINEARITIES AT INFINITY
}

\author{
Guanwei Chen
}

\begin{abstract}
By using variational methods and critical point theory, in particular, a generalized weak linking theorem, we study a first order nonlinear Hamiltonian system with asymptotically linear nonlinearity at infinity. We obtain the existence of ground state homoclinic orbits for this nonlinear Hamiltonian system. In particular, we obtain a necessary and sufficient condition for the existence of ground state homoclinic orbits. To the best of our knowledge, there is no published result focusing on necessary and sufficient conditions of the existence of ground state homoclinic orbits for this system.
\end{abstract}

\section{Introduction and main results}

In this paper, we consider the following first order nonlinear Hamiltonian system:

$$
-J \dot{u}(t)-L(t) u=\nabla W(t, u(t)), \quad t \in \mathbb{R},
$$

where $J=\left(\begin{array}{cc}0 & -I_{n} \\ I_{n} & 0\end{array}\right)$ denotes the standard symplectic matrix, $L(t)$ is a given continuous $T$-periodic and symmetric $2 N \times 2 N$-matrix-value function and $W(t, u) \in$

2010 Mathematics Subject Classification. 37J45, 37K05, 58E05.

Key words and phrases. Nonlinear Hamiltonian system; necessary and sufficient condition; ground state homoclinic orbit; asymptotically linear; generalized weak linking theorem.

Research supported by the National Natural Science Foundation of China (No. 11401011, No. 11326113). 
$C^{1}\left(\mathbb{R} \times \mathbb{R}^{2 N}, \mathbb{R}\right)$ is $T$-periodic in the $t$-variable and $\nabla W(t, u)$ denotes its gradient with respect to the $u$ variable. Recall that a solution $u$ of (1.1) is a homoclinic orbit if $u \neq 0$ and $u \rightarrow 0$ as $|t| \rightarrow \infty$.

In this paper, we are interested in the strongly indefinite case for (1.1), that is,

$\left(\mathrm{A}_{1}\right) \underline{\Lambda}:=\sup (\sigma(B) \cap(-\infty, 0))<0<\bar{\Lambda}:=\inf (\sigma(B) \cap(0, \infty))$, where $B:=$ $-J \frac{d}{d t^{2}}-L(t)$ and $\sigma(B)$ denotes the spectrum of $B$. Clearly, $\sigma(B)$ is absolutely continuous.

REMARK 1.1. If $\left(A_{1}\right)$ holds and the nonlinearity $W(t, u)$ of $(1.1)$ is $s u$ perquadratic at infinity, i.e.

$$
\lim _{|u| \rightarrow \infty} \frac{W(t, u)}{|u|^{2}}=+\infty
$$

the authors of [2] have obtained the existence of ground state homoclinic orbits of (1.1) (i.e. solutions corresponding to the least energy of the action functional of (1.1)). Inspired by [2], we study the existence of ground state homoclinic orbits of $(1.1)$ in the case where $W(t, u)$ is asymptotically quadratic at infinity, see Theorem 1.3. As we know, the asymptotically quadratic case is very different from the superquadratic case. In particular, we obtain a necessary and sufficient condition of the existence of ground state homoclinic orbits for (1.1), see Theorem 1.4.

REMARK 1.2. The main novelty of this paper is that we obtain a necessary and sufficient condition for the existence of ground state homoclinic orbits in the strongly indefinite case $\left(A_{1}\right)$, see Theorem 1.4. In fact, for the positive definite case, i.e. $\inf \sigma(B)>0$, we believe that the necessary and sufficient condition can also be obtained.

Let $\widetilde{W}(t, u):=(\nabla W(t, u), u) / 2-W(t, u)$, where $(\cdot, \cdot)$ denotes the standard inner product in $\mathbb{R}^{2 N}$, and the associated norm is denoted by $|\cdot|$. We assume that

$\left(\mathrm{W}_{1}\right)|\nabla W(t, u)|=o(|u|)$ as $|u| \rightarrow 0$ uniformly in $t \in \mathbb{R}$.

$\left(\mathrm{W}_{2}\right) W(t, u) \geq 0$ for all $(t, u) \in \mathbb{R} \times \mathbb{R}^{2 N}$ and $\widetilde{W}(t, u)>0$ if $u \in \mathbb{R}^{2 N} \backslash\{0\}$.

$\left(\mathrm{W}_{3}\right) W(t, u)=V(t)|u|^{2} / 2+F(t, u)$, where $0<V(t)<+\infty$ and

$$
|\nabla F(t, u)|=o(|u|) \quad \text { as }|u| \rightarrow \infty \text { uniformly in } t .
$$

$\left(\mathrm{W}_{4}\right) \widetilde{W}(t, u) \rightarrow+\infty$ as $|u| \rightarrow+\infty$, and there is a function $P(t)(|P(t)|<+\infty$, for all $t \in \mathbb{R}$ ) such that

$$
\limsup _{|u| \rightarrow 0} \frac{|\nabla W(t, u)|^{2}}{\widetilde{W}(t, u)}=P(t) \quad \text { uniformly in } t .
$$

Now, our main results read as follows: 
TheOREM 1.3. If $\left(\mathrm{A}_{1}\right)$ and $\left(\mathrm{W}_{1}\right)-\left(\mathrm{W}_{4}\right)$ hold, and $V(t)>\bar{\Lambda}$ for all $t \in \mathbb{R}$, then (1.1) has at least one nontrivial homoclinic orbit, which is a ground state solution.

As we know, nonlinear problems usually do not have necessary and sufficient conditions of the existence of solutions. However, we obtain a necessary and sufficient condition of the existence of ground state homoclinic orbit for (1.1).

Theorem 1.4. Assume that $\left(\mathrm{A}_{1}\right)$ and $\left(\mathrm{W}_{1}\right)-\left(\mathrm{W}_{4}\right)$ with $V(t) \equiv V$ in $\left(\mathrm{W}_{3}\right)$ hold ( $V$ is a positive constant). If $|\nabla W(t, u)| /|u| \leq V$, for all $(t, u) \in \mathbb{R} \times \mathbb{R}^{2 N}$, and

$$
\underline{\Lambda}+V \leq \min \{0, \bar{\Lambda}-V\},
$$

then (1.1) admits a nontrivial homoclinic orbit if and only if $V>\bar{\Lambda}$.

REMARK 1.5. Notice that (1.2) always holds if $V$ is small enough, thus Theorem 1.4 shows that $V>\bar{\Lambda}$ is a sharp condition for the existence of ground state homoclinic orbit for (1.1). To the best of our knowledge, there is no published result focusing on necessary and sufficient conditions of the existence of ground state homoclinic orbits for (1.1).

Next, we give an example, which illustrates the main results.

EXAMPLE 1.6. Let

$$
W(t, u):=\frac{1}{2} V(t)\left(|u|^{2}-\ln \left(1+|u|^{2}\right)\right),
$$

where $0<V(t)<+\infty$ is continuous and $T$-periodic. It is not hard to check that it satisfies conditions $\left(\mathrm{W}_{1}\right)-\left(\mathrm{W}_{4}\right)$.

It is maybe worthwhile recalling here that since appearance in 1990 of the work [3], there were obtained many sufficient conditions of the existence of homoclinic orbits for systems (1.1). For example, in the papers [1], [3], [6]-[8], [10], $[11],[13],[15],[16]$ there were obtained sufficient conditions of the existence of homoclinic orbits for (1.1) by considering the well-known Ambrosetti-Rabinowitz (AR for short) super quadratic condition. Roughly speaking the role of AR super quadratic condition is to insure that all Palais-Smale sequences for the corresponding function of (1.1) at the Mountain-Pass level are bounded. If $W(t, u)$ and $L(t)$ depend periodically on $t$ so that 0 lies in a gap of $\sigma\left(-J \frac{d}{d t}-L\right)$, Ding [4] has obtained sufficient conditions of the existence of infinitely many homoclinic orbits for $(1.1)$ with $W(t, u)$ super quadratic or asymptotically quadratic in $u$ at infinity by some recent information on strongly indefinite functionals in critical point theory. If $W(t, u)$ and $L(t)$ depend periodically on $t$ while 0 lies on the boundary of $\sigma\left(-J \frac{d}{d t}-L\right)$, Ding and Willem [7] have obtained sufficient conditions of the existence of one homoclinic orbit, and Ding and Girardi [6] have 
got sufficient conditions of the existence of infinitely many homoclinic orbits for some super quadratic systems. The authors of [3] firstly studied sufficient conditions of the existence of homoclinic orbits for (1.1) by means of critical point theory. Under the assumptions that $L$ is constant and 0 is a hyperbolic point of $-\left(J \frac{d}{d t}+L\right), W(t, u)$ strictly convex in $u$ and satisfying the AR super quadratic condition, they obtained the existence and multiplicity of homoclinic orbits of (1.1). This result was deepened in [10], [11] when Séré established the existence of infinitely many homoclinic orbits. Independently, Hofer and Wysocki [8], using Fredholm operator theory and a linking argument, and Tanaka [13], passing through a subharmonic approach, managed to remove the convexity assumption to get sufficient conditions of the existence of one homoclinic orbit. Later linking type arguments were used in [1], [6], [7] to get sufficient conditions of the existence and multiplicity of homoclinic orbits of (1.1) when $L$ depend periodically on $t$ and certain symmetries on $W(t, u)$ are assumed for the multiplicity.

The rest of this paper is organized as follows. In Section 2, we first establish the variational framework of (1.1), and then we give some preliminary lemmas, which are useful in the proofs of our main results. In Section 3, we give the detailed proofs of our main results.

\section{Variational framework and preliminary lemmas}

We first give some notations used in this paper.

Notations. Let $B_{r}(s):=[s-r, s+r]$. Let $\|\cdot\|_{L^{q}}$ and $(\cdot, \cdot)_{L^{q}}$ denote the usual norm and the inner product of $L^{q}\left(\mathbb{R}, \mathbb{R}^{2 N}\right)$, respectively.

Under assumption $\left(\mathrm{A}_{1}\right), B=-J \frac{d}{d t}-L(t)$ is a self-adjoint operator acting on $L^{2}\left(\mathbb{R}, \mathbb{R}^{2 N}\right)$ with the domain $\mathcal{D}(B):=H^{1}\left(\mathbb{R}, \mathbb{R}^{2 N}\right)$. Let $E:=\mathcal{D}\left(|B|^{1 / 2}\right)$ be the domain of the self-adjoint operator $|B|^{1 / 2}$ which is a Hilbert space equipped with the inner product

$$
\langle u, v\rangle_{\mathcal{D}}:=(u, v)_{L^{2}}+\left(|B|^{1 / 2} u,|B|^{1 / 2} v\right)_{L^{2}} .
$$

Moreover, to $B$ there corresponds a bounded self-adjoint operator $\chi: E \rightarrow E$ such that

$$
\langle\chi u, v\rangle_{\mathcal{D}}=\int_{\mathbb{R}}(-J \dot{u}-L(t) u, v) d t
$$

$E=E^{-} \oplus E^{+}$, where $E^{ \pm}$are $\chi$-invariant and $\left\langle u^{+}, u^{-}\right\rangle_{\mathcal{D}}=\left(u^{+}, u^{-}\right)_{L^{2}}=0$, for all $u^{ \pm} \in E^{ \pm}$. Also, $\langle\chi u, u\rangle_{\mathcal{D}}$ is positive definite on $E^{+}$and negative definite on $E^{-}$. We introduce a new inner product in $E$ by setting

$$
\langle u, v\rangle:=\left\langle\chi u^{+}, v^{+}\right\rangle_{\mathcal{D}}-\left\langle\chi u^{-}, v^{-}\right\rangle_{\mathcal{D}}
$$

Then $\langle\chi u, u\rangle_{\mathcal{D}}=\left\|u^{+}\right\|^{2}-\left\|u^{-}\right\|^{2}$, where $\|\cdot\|$ is the norm corresponding to $\langle\cdot, \cdot\rangle$. Obviously, the decomposition $E=E^{-} \oplus E^{+}$is orthogonal with respect to both 
$\langle\cdot, \cdot\rangle$ and $(\cdot, \cdot)_{L^{2}}$. Therefore, the corresponding functional with (1.1) can be rewritten as:

$$
\begin{aligned}
I(u) & =\frac{1}{2} \int_{\mathbb{R}}(-J \dot{u}-L(t) u, u) d t-\int_{\mathbb{R}} W(t, u) d t \\
& =\frac{1}{2}\left(\left\|u^{+}\right\|^{2}-\left\|u^{-}\right\|^{2}\right)-\int_{\mathbb{R}} W(t, u) d t .
\end{aligned}
$$

The hypotheses on $W$ imply that $I \in C^{1}(E, \mathbb{R})$ and a standard argument shows that critical points of $I$ are homoclinic orbits of (1.1).

The following abstract critical point theorem plays an important role in proving our main result. Let $E$ be a Hilbert space with norm $\|\cdot\|$ and have an orthogonal decomposition $E=N \oplus N^{\perp}, N \subset E$ is a closed and separable subspace. There exists norm $|v|_{\omega}$ satisfying $|v|_{\omega} \leq\|v\|$ for all $v \in N$ and inducing a topology equivalent to the weak topology of $N$ on bounded subset of $N$. For $u=v+w \in E=N \oplus N^{\perp}$ with $v \in N, w \in N^{\perp}$, we define $|u|_{\omega}^{2}=|v|_{\omega}^{2}+\|w\|^{2}$. Particularly, if $\left(u_{n}=v_{n}+w_{n}\right)$ is $\|\cdot\|$-bounded and $u_{n} \stackrel{\left.|\cdot|\right|_{\omega}}{\longrightarrow} u$, then $v_{n} \rightarrow v$ weakly in $N, w_{n} \rightarrow w$ strongly in $N^{\perp}, u_{n} \rightarrow v+w$ weakly in $E$ (cf. [12]).

Let $E=E^{-} \oplus E^{+}, z_{0} \in E^{+}$with $\left\|z_{0}\right\|=1$. Let $N:=E^{-} \oplus R z_{0}$ and $E_{1}^{+}:=N^{\perp}=\left(E^{-} \oplus R z_{0}\right)^{\perp}$. For $R>0$, let

$$
Q:=\left\{u:=u^{-}+s z_{0} \mid s \in \mathbb{R}^{+}, u^{-} \in E^{-},\|u\|<R\right\}
$$

with $p_{0}=s_{0} z_{0} \in Q, s_{0}>0$. We define

$$
D:=\left\{u:=s z_{0}+w^{+} \mid s \in \mathbb{R}, w^{+} \in E_{1}^{+},\left\|s z_{0}+w^{+}\right\|=s_{0}\right\} .
$$

For $I \in C^{1}(E, \mathbb{R})$, define $\Gamma:=\left\{h \mid h:[0,1] \times \bar{Q} \mapsto E\right.$ is $|\cdot|{ }_{\omega}$-continuous, $h(0, u)=u, I(h(s, u)) \leq I(u)$, for all $u \in \bar{Q}$. For any $\left(s_{0}, u_{0}\right) \in[0,1] \times \bar{Q}$, there is a $|\cdot|_{\omega}$-neighbourhood $U_{\left(s_{0}, u_{0}\right)}$, such that

$$
\left.\left\{u-h(t, u) \mid(t, u) \in U_{\left(s_{0}, u_{0}\right)} \cap([0,1] \times \bar{Q})\right\} \subset E_{\text {fin }}\right\},
$$

where $E_{\text {fin }}$ denotes various finite-dimensional subspaces of $E, \Gamma \neq 0$ since id $\in \Gamma$. The weak linking variant is:

Lemma 2.1 ([12]). The family of $C^{1}$-functionals $\left\{I_{\lambda}\right\}$ has the form $I_{\lambda}(u):=$ $J(u)-\lambda K(u)$ for all $\lambda \in[1,2]$. Assume that:

(a) $K(u) \geq 0$, for all $u \in E, I_{1}=I$;

(b) $J(u) \rightarrow \infty$ or $K(u) \rightarrow \infty$ as $\|u\| \rightarrow \infty$;

(c) $I_{\lambda}$ is $|\cdot|_{\omega}$-upper semicontinuous, $I_{\lambda}^{\prime}$ is weakly sequentially continuous on E. Moreover, $I_{\lambda}$ maps bounded sets to bounded sets;

(d) $\sup _{\partial Q} I_{\lambda}<\inf _{D} I_{\lambda}$, for all $\lambda \in[1,2]$.

Then, for almost all $\lambda \in[1,2]$, there exists a sequence $\left\{u_{n}\right\}$ such that

$$
\sup _{n}\left\|u_{n}\right\|<\infty, \quad I_{\lambda}^{\prime}\left(u_{n}\right) \rightarrow 0, \quad I_{\lambda}\left(u_{n}\right) \rightarrow c_{\lambda},
$$


where $c_{\lambda}:=\inf _{h \in \Gamma} \sup _{u \in Q} I_{\lambda}(h(1, u)) \in\left[\inf _{D} I_{\lambda}, \sup _{\bar{Q}} I\right]$.

In order to apply Lemma 2.1, we consider

$$
I_{\lambda}(u):=\frac{1}{2}\left\|u^{+}\right\|^{2}-\lambda\left(\frac{1}{2}\left\|u^{-}\right\|^{2}+\int_{\mathbb{R}} W(t, u) d t\right) .
$$

It is easy to see that $I_{\lambda}$ satisfies conditions (a), (b) in Lemma 2.1. To see (c), if $u_{n} \stackrel{|\cdot|_{\omega}}{\longrightarrow} u$ and $I_{\lambda}\left(u_{n}\right) \geq a$, then $u_{n}^{+} \rightarrow u^{+}$and $u_{n}^{-} \rightarrow u^{-}$in $E$, going to a subsequence if necessary, $u_{n} \rightarrow u$ almost everywhere on $\mathbb{R}$. Next, we prove $I_{\lambda}(u) \geq a$, which means that $I_{\lambda}$ is $|\cdot|_{\omega}$-upper semicontinuous. Since

$$
I_{\lambda}\left(u_{n}\right)=\frac{1}{2}\left\|u_{n}^{+}\right\|^{2}-\lambda\left(\frac{1}{2}\left\|u_{n}^{-}\right\|^{2}+\int_{\mathbb{R}} W\left(t, u_{n}\right) d t\right) \geq a,
$$

it follows from $u_{n}^{+} \rightarrow u^{+}$and $u_{n}^{-} \rightarrow u^{-}$in $E$, the weak lower semicontinuity of the norm, $W\left(t, u_{n}\right) \geq 0$ and the Fatou's lemma that

$$
\begin{aligned}
a \leq \limsup _{n \rightarrow \infty} I_{\lambda}\left(u_{n}\right) & =\limsup _{n \rightarrow \infty}\left[\frac{1}{2}\left\|u_{n}^{+}\right\|^{2}-\lambda\left(\frac{1}{2}\left\|u_{n}^{-}\right\|^{2}+\int_{\mathbb{R}} W\left(t, u_{n}\right) d t\right)\right] \\
& \leq \frac{1}{2}\left\|u^{+}\right\|^{2}-\liminf _{n \rightarrow \infty} \lambda\left(\frac{1}{2}\left\|u_{n}^{-}\right\|^{2}+\int_{\mathbb{R}} W\left(t, u_{n}\right) d t\right) \\
& \leq \frac{1}{2}\left\|u^{+}\right\|^{2}-\lambda\left(\frac{1}{2}\left\|u^{-}\right\|^{2}+\int_{\mathbb{R}} W(t, u) d t\right)=I_{\lambda}(u) .
\end{aligned}
$$

Thus we get $I_{\lambda}(u) \geq a$. $I_{\lambda}^{\prime}$ is weakly sequentially continuous on $E$, see [14].

Lemma $2.2([5,15])$. E embeds continuously into $L^{q}\left(\mathbb{R}, \mathbb{R}^{2 N}\right)$ and compactly into $L_{\mathrm{loc}}^{q}\left(\mathbb{R}, \mathbb{R}^{2 N}\right)$ for all $q \in[2, \infty)$.

In this section, we always assume $\left(\mathrm{A}_{1}\right)$ and $\left(\mathrm{W}_{1}\right)-\left(\mathrm{W}_{4}\right)$ hold, and $V(t)>\bar{\Lambda}$ for all $t \in \mathbb{R}$. To use Lemma 2.1, we still need to verify condition (d). Indeed, we have:

LEMma 2.3. The following facts hold true:

(a) There exists $\rho>0$ independent of $\lambda \in[1,2]$ such that $\kappa:=\inf I_{\lambda}\left(S_{\rho} E^{+}\right)$ $>0$, where $S_{\rho} E^{+}:=\left\{z \in E^{+} \mid\|z\|=\rho\right\}$.

(b) For fixed $z_{0} \in E^{+}$with $\left\|z_{0}\right\|=1$ and any $\lambda \in[1,2]$, there is $R>\rho>0$ such that $\sup I_{\lambda}(\partial Q) \leq 0$, where $Q:=\left\{u:=v+s z_{0} \mid s \geq 0, v \in E^{-}\right.$, $\|u\|<R\}$.

Proof. (a) By $\left(\mathrm{W}_{1}\right)$ and $\left(\mathrm{W}_{3}\right)$, for any $\varepsilon>0$ there exists $C_{\varepsilon}>0$ such that

$$
|\nabla W(t, u)| \leq \varepsilon|u|+C_{\varepsilon}|u|^{p-1}, \quad|W(t, u)| \leq \varepsilon|u|^{2}+C_{\varepsilon}|u|^{p},
$$

where $p>2$. Hence, by Lemma 2.2 , for any $u \in E^{+}$, we have

$$
I_{\lambda}(u) \geq \frac{1}{2}\|u\|^{2}-\lambda \varepsilon\|u\|^{2}-C_{\varepsilon}^{\prime}\|u\|^{p} .
$$

It implies the conclusion. 
(b) Note that $V(t)$ is $T$-periodic, it follows from $V(t)>\bar{\Lambda}$ for all $t \in \mathbb{R}$ that there exists $\varepsilon_{0}>0$ such that $V(t) \geq \bar{\Lambda}+2 \varepsilon_{0}$ for all $t \in \mathbb{R}$. Note that $\sigma(B)$ is absolutely continuous, so we can choose $z_{0} \in E^{+}$with $\left\|z_{0}\right\|=1$ such that $\left\|z_{0}\right\|^{2} \leq\left(\bar{\Lambda}+\varepsilon_{0}\right)\left\|z_{0}\right\|_{L^{2}}^{2}$.

Suppose by contradiction that there exist $u_{n} \in E^{-} \oplus \mathbb{R}^{+} z_{0}$ such that $I_{\lambda}\left(u_{n}\right)>0$ for all $n$ and $\left\|u_{n}\right\| \rightarrow \infty$ as $n \rightarrow \infty$. Set $w_{n}=u_{n} /\left\|u_{n}\right\|=s_{n} z_{0}+w_{n}^{-}$, then

$$
0<\frac{I_{\lambda}\left(u_{n}\right)}{\left\|u_{n}\right\|^{2}}=\frac{1}{2}\left(s_{n}^{2}-\lambda\left\|w_{n}^{-}\right\|^{2}\right)-\lambda \int_{\mathbb{R}} \frac{W\left(t, u_{n}\right)}{\left|u_{n}\right|^{2}}\left|w_{n}\right|^{2} d t .
$$

From $\left(\mathrm{W}_{2}\right)$, we know $W(t, u) \geq 0$ and have $\left\|w_{n}^{-}\right\|^{2} \leq \lambda\left\|w_{n}^{-}\right\|^{2}<s_{n}^{2}=1-\left\|w_{n}^{-}\right\|^{2}$, therefore, $\left\|w_{n}^{-}\right\| \leq 1 / \sqrt{2}$ and $1 / \sqrt{2} \leq s_{n} \leq 1$. So $s_{n} \rightarrow s \neq 0$ after passing to a subsequence, $w_{n} \rightarrow w$ and $w_{n} \rightarrow w$ almost everywhere in $\mathbb{R}$. Hence $w=$ $s z_{0}+w^{-} \neq 0$, and thus $\left|u_{n}\right|=\left|w_{n}\right| \cdot\left\|u_{n}\right\| \rightarrow \infty$. It follows from $\left(\mathrm{W}_{2}\right),\left(\mathrm{W}_{3}\right)$, (2.4), $\left\|z_{0}\right\|=1, V(t) \geq \bar{\Lambda}+2 \varepsilon_{0}$ for all $t \in \mathbb{R},\left\|z_{0}\right\|^{2} \leq\left(\bar{\Lambda}+\varepsilon_{0}\right)\left\|z_{0}\right\|_{L^{2}}^{2}$, Fatou's lemma and the weak lower semicontinuity of the norm that

$$
\begin{aligned}
0 & \leq \limsup _{n \rightarrow \infty} \frac{I_{\lambda}\left(u_{n}\right)}{\left\|u_{n}\right\|^{2}} \\
& =\limsup _{n \rightarrow \infty}\left(\frac{1}{2}\left(s_{n}^{2}-\lambda\left\|w_{n}^{-}\right\|^{2}\right)-\lambda \int_{\mathbb{R}} \frac{W\left(t, u_{n}\right)}{\left|u_{n}\right|^{2}}\left|w_{n}\right|^{2} d t\right) \\
& \leq \frac{1}{2}\left(s^{2}\left\|z_{0}\right\|^{2}-\left\|w^{-}\right\|^{2}\right)-\frac{1}{2} \int_{\mathbb{R}} V(t) w^{2} d t \\
& \leq \frac{1}{2} s^{2}\left\|z_{0}\right\|^{2}-\frac{1}{2}\left(\bar{\Lambda}+2 \varepsilon_{0}\right) s^{2}\left\|z_{0}\right\|_{L^{2}}^{2} \\
& \leq \frac{1}{2} s^{2}\left(\bar{\Lambda}+\varepsilon_{0}\right)\left\|z_{0}\right\|_{L^{2}}^{2}-\frac{1}{2}\left(\bar{\Lambda}+2 \varepsilon_{0}\right) s^{2}\left\|z_{0}\right\|_{L^{2}}^{2}=-\frac{1}{2} \varepsilon_{0} s^{2}\left\|z_{0}\right\|_{L^{2}}^{2}<0,
\end{aligned}
$$

which is a contradiction. Therefore, the proof is finished.

Lemma 2.4. For almost all $\lambda \in[1,2]$, there exists $u_{\lambda}$ such that $I_{\lambda}^{\prime}\left(u_{\lambda}\right)=0$ and $I_{\lambda}\left(u_{\lambda}\right) \leq \sup _{\bar{Q}} I$.

Proof. By Lemmas 2.1 and 2.3, for almost all $\lambda \in[1,2]$, there exists a sequence $\left\{u_{n}\right\}$ such that $\sup _{n}\left\|u_{n}\right\|<\infty, I_{\lambda}^{\prime}\left(u_{n}\right) \rightarrow 0$ and $I_{\lambda}\left(u_{n}\right) \rightarrow c_{\lambda} \in\left[\kappa, \sup _{\bar{Q}} I\right]$, where $\kappa$ is defined in Lemma 2.3.

We write $u_{n}=u_{n}^{-}+u_{n}^{+}$with $u_{n}^{ \pm} \in E^{ \pm}$. Since $\left\{u_{n}^{+}\right\}$is bounded, by a Lion's concentration compactness principle [9], either $\left\{u_{n}^{+}\right\}$is vanishing: for each $l>0$,

$$
\lim _{n \rightarrow \infty} \sup _{s \in \mathbb{R}} \int_{B_{l}(s)}\left|u_{n}^{+}\right|^{2} d t=0
$$

(in this case $u_{n}^{+} \rightarrow 0$ in $L^{q}\left(\mathbb{R}, \mathbb{R}^{2 N}\right)$ for all $q \in(2, \infty)$ ), or it is non-vanishing: there exist $r, \delta>0$ and a sequence $s_{n} \in \mathbb{R}$ such that

$$
\lim _{n \rightarrow \infty} \int_{B_{r}\left(s_{n}\right)}\left|u_{n}^{+}\right|^{2} d t \geq \delta
$$


If $\left\{u_{n}^{+}\right\}$is vanishing, then $u_{n}^{+} \rightarrow 0$ in $L^{q}\left(\mathbb{R}, \mathbb{R}^{2 N}\right)$ for all $q \in(2, \infty)$, it follows from (2.3), the boundedness of $\left\{u_{n}\right\}$ and Hölder's inequality that

$$
\begin{aligned}
\int_{\mathbb{R}}\left|\left(\nabla W\left(t, u_{n}\right), u_{n}^{+}\right)\right| d t & \leq \varepsilon \int_{\mathbb{R}}\left|u_{n}\right| \cdot\left|u_{n}^{+}\right| d t+C_{\varepsilon} \int_{\mathbb{R}}\left|u_{n}\right|^{p-1}\left|u_{n}^{+}\right| d t \\
& \leq \varepsilon\left\|u_{n}\right\|_{L^{2}}\left\|u_{n}^{+}\right\|_{L^{2}}+C_{\varepsilon}\left\|u_{n}\right\|_{L^{p}}^{p-1}\left\|u_{n}^{+}\right\|_{L^{p}} \rightarrow 0
\end{aligned}
$$

as $n \rightarrow \infty$. Therefore,

$$
I_{\lambda}\left(u_{n}\right) \leq\left\|u_{n}^{+}\right\|^{2}=I_{\lambda}^{\prime}\left(u_{n}\right) u_{n}^{+}+\lambda \int_{\mathbb{R}}\left(\nabla W\left(t, u_{n}\right), u_{n}^{+}\right) d t \rightarrow 0
$$

as $n \rightarrow \infty$, which contradicts with the fact that $I_{\lambda}\left(u_{n}\right) \geq \kappa$. Hence $\left\{u_{n}^{+}\right\}$must be non-vanishing. Let us define $v_{n}=u_{n}\left(\cdot-s_{n}\right)$, then

$$
\lim _{n \rightarrow \infty} \int_{B_{r}(0)}\left|v_{n}^{+}\right|^{2} d t \geq \frac{\delta}{2} .
$$

Since $I_{\lambda}$ and $I_{\lambda}^{\prime}$ are both invariant under translation, we know that $I_{\lambda}^{\prime}\left(v_{n}\right) \rightarrow 0$ and $I_{\lambda}\left(v_{n}\right) \rightarrow c_{\lambda}$ as $n \rightarrow \infty$. Since $\left\{v_{n}\right\}$ is still bounded, we may assume that $v_{n}^{+} \rightarrow u_{\lambda}^{+}, v_{n}^{-} \rightarrow u_{\lambda}^{-}$in $E$. Lemma 2.2 implies $v_{n}^{+} \rightarrow u_{\lambda}^{+}$in $L_{\text {loc }}^{2}\left(\mathbb{R}, \mathbb{R}^{2 N}\right)$, it follows from (2.5) that $u_{\lambda}=u_{\lambda}^{+}+u_{\lambda}^{-} \neq 0$ and $I_{\lambda}^{\prime}\left(u_{\lambda}\right) \varphi=\lim _{n \rightarrow \infty} I_{\lambda}^{\prime}\left(v_{n}\right) \varphi=0$, for all $\varphi \in C_{0}^{\infty}(\mathbb{R})$. By $\left(\mathrm{W}_{2}\right)$ and Fatou's lemma, we have

$$
\begin{aligned}
\sup _{\bar{Q}} I \geq c_{\lambda} & =\lim _{n \rightarrow \infty}\left(I_{\lambda}\left(v_{n}\right)-\frac{1}{2} I_{\lambda}^{\prime}\left(v_{n}\right) v_{n}\right) \\
& =\lim _{n \rightarrow \infty} \int_{\mathbb{R}}\left(\frac{1}{2}\left(\nabla W\left(t, v_{n}\right), v_{n}\right)-W\left(t, v_{n}\right)\right) d t \\
& \geq \int_{\mathbb{R}}\left(\frac{1}{2}\left(\nabla W\left(t, u_{\lambda}\right), u_{\lambda}\right)-W\left(t, u_{\lambda}\right)\right) d t=I_{\lambda}\left(u_{\lambda}\right),
\end{aligned}
$$

thus we get $I_{\lambda}\left(u_{\lambda}\right) \leq \sup _{\bar{Q}} I$.

Lemma 2.5. There exist $\left\{\lambda_{n}\right\} \subset[1,2]$ with $\lambda_{n} \rightarrow 1$ and $\left\{u_{\lambda_{n}}\right\}$ such that $I_{\lambda_{n}}^{\prime}\left(u_{\lambda_{n}}\right)=0$ and $I_{\lambda_{n}}\left(u_{\lambda_{n}}\right) \leq \sup _{\bar{Q}} I$. Moreover, $\left\{u_{\lambda_{n}}\right\}$ is bounded.

Proof. The existence of $\left\{\lambda_{n}\right\} \subset[1,2]$ with $\lambda_{n} \rightarrow 1$ and $\left\{u_{\lambda_{n}}\right\}$ such that $I_{\lambda_{n}}^{\prime}\left(u_{\lambda_{n}}\right)=0$ and $I_{\lambda_{n}}\left(u_{\lambda_{n}}\right) \leq \sup _{\bar{Q}} I$ is the direct consequence of Lemma 2.4.

The facts $I_{\lambda_{n}}^{\prime}\left(u_{\lambda_{n}}\right)=0$ and $I_{\lambda_{n}}\left(u_{\lambda_{n}}\right) \leq \sup _{\bar{Q}} I$ imply that

$$
\frac{1}{\lambda_{n}}\left(I_{\lambda_{n}}\left(u_{\lambda_{n}}\right)-\frac{1}{2} I_{\lambda_{n}}^{\prime}\left(u_{\lambda_{n}}\right) u_{\lambda_{n}}\right) \leq C,
$$

it follows from the definition of $I_{\lambda_{n}}$ that

$$
\int_{\mathbb{R}} \widetilde{W}\left(t, u_{\lambda_{n}}\right) d t=\frac{1}{\lambda_{n}}\left(I_{\lambda_{n}}\left(u_{\lambda_{n}}\right)-\frac{1}{2} I_{\lambda_{n}}^{\prime}\left(u_{\lambda_{n}}\right) u_{\lambda_{n}}\right) \leq C .
$$


Note that $\left(\mathrm{W}_{4}\right)$ implies $\int_{\mathbb{R}} \widetilde{W}(t, u) d t \rightarrow+\infty$ as $|u| \rightarrow+\infty$, thus it follows from (2.6) that

$$
\left|u_{\lambda_{n}}\right| \leq C_{1}
$$

for some positive constant $C_{1}$. From $\left(\mathrm{W}_{4}\right)$, there exists a positive constants $C_{2}$ such that

$$
\left|\nabla W\left(t, u_{\lambda_{n}}\right)\right|^{2} \leq C_{2} \widetilde{W}\left(t, u_{\lambda_{n}}\right), \quad t \in \mathbb{R} \text { and }\left|u_{\lambda_{n}}\right| \leq C_{1}
$$

Thus by $(2.6)-(2.8)$, the fact $I_{\lambda_{n}}^{\prime}\left(u_{\lambda_{n}}\right) u_{\lambda_{n}}^{+}=0$, Hölder's inequality and the Sobolev imbedding theorem,

$$
\begin{aligned}
\left\|u_{\lambda_{n}}^{+}\right\|^{2} & =\lambda_{n} \int_{\mathbb{R}}\left(\nabla W\left(t, u_{\lambda_{n}}\right), u_{\lambda_{n}}^{+}\right) d t \\
& \leq \lambda_{n}\left(\int_{\mathbb{R}}\left|\nabla W\left(t, u_{\lambda_{n}}\right)\right|^{2} d t\right)^{1 / 2}\left(\int_{\mathbb{R}}\left|u_{\lambda_{n}}^{+}\right|^{2} d t\right)^{1 / 2} \\
& \leq C_{3}\left(\int_{\mathbb{R}}\left|\nabla W\left(t, u_{\lambda_{n}}\right)\right|^{2} d t\right)^{1 / 2}\left\|u_{\lambda_{n}}^{+}\right\| \leq C_{4}\left\|u_{\lambda_{n}}^{+}\right\|
\end{aligned}
$$

for some positive constants $C_{3}$ and $C_{4}$. It implies that $\left\|u_{\lambda_{n}}^{+}\right\| \leq C$. On the other hand, the condition $\left(\mathrm{W}_{2}\right)$ and $I_{\lambda_{n}}^{\prime}\left(u_{\lambda_{n}}\right) u_{\lambda_{n}}=0$ imply that

$$
\left\|u_{\lambda_{n}}^{+}\right\|^{2}-\lambda_{n}\left\|u_{\lambda_{n}}^{-}\right\|^{2}=\lambda_{n} \int_{\mathbb{R}}\left(\nabla W\left(t, u_{\lambda_{n}}\right), u_{\lambda_{n}}\right) d t \geq 0
$$

that is, $\left\|u_{\lambda_{n}}^{-}\right\|^{2} \leq \lambda_{n}\left\|u_{\lambda_{n}}^{-}\right\|^{2} \leq\left\|u_{\lambda_{n}}^{+}\right\|^{2}$. Therefore, we get $\left\{u_{\lambda_{n}}\right\}$ is bounded.

LEMMA 2.6. If $\left\{u_{\lambda_{n}}\right\}$ is the sequence obtained in Lemma 2.5, then it is also $a$ (PS) sequence for $I$ satisfying $\lim _{n \rightarrow \infty} I^{\prime}\left(u_{\lambda_{n}}\right)=0$ and $\lim _{n \rightarrow \infty} I\left(u_{\lambda_{n}}\right) \leq \sup _{\bar{Q}} I$.

Proof. Note that $u_{\lambda_{n}}$ is bounded. From

$$
\lim _{n \rightarrow \infty} I\left(u_{\lambda_{n}}\right)=\lim _{n \rightarrow \infty}\left(I_{\lambda_{n}}\left(u_{\lambda_{n}}\right)+\left(\lambda_{n}-1\right)\left(\frac{1}{2}\left\|u_{\lambda_{n}}^{-}\right\|^{2}+\int_{\mathbb{R}} W\left(t, u_{\lambda_{n}}\right), d t\right)\right)
$$

and

$$
\begin{aligned}
& \lim _{n \rightarrow \infty} I^{\prime}\left(u_{\lambda_{n}}\right) \varphi \\
& \quad=\lim _{n \rightarrow \infty}\left(I_{\lambda_{n}}^{\prime}\left(u_{\lambda_{n}}\right) \varphi+\left(\lambda_{n}-1\right)\left(\left\langle u_{\lambda_{n}}^{-}, \varphi^{-}\right\rangle+\int_{\mathbb{R}}\left(\nabla W\left(t, u_{\lambda_{n}}\right), \varphi\right) d t\right)\right)
\end{aligned}
$$

for any $\varphi \in E$, we obtain the conclusion. 


\section{Proofs of main results}

Proof of TheOrem 1.3. Lemma 2.5 implies $\left\{u_{\lambda_{n}}\right\}$ is bounded, thus we have either $\left\{u_{\lambda_{n}}\right\}$ is vanishing: for each $l>0$,

$$
\lim _{n \rightarrow \infty} \sup _{s \in \mathbb{R}} \int_{B_{l}(s)}\left|u_{\lambda_{n}}\right|^{2} d t=0
$$

or non-vanishing: there exist $r, \delta>0$ and a sequence $\left\{s_{n}\right\} \subset \mathbb{R}$ such that

$$
\lim _{n \rightarrow \infty} \int_{B_{r}\left(s_{n}\right)}\left|u_{\lambda_{n}}\right|^{2} d t \geq \delta
$$

If $\left\{u_{\lambda_{n}}\right\}$ is vanishing, Lion's concentration compactness principle implies $u_{\lambda_{n}} \rightarrow$ 0 in $L^{p}\left(\mathbb{R}, \mathbb{R}^{2 N}\right)$, for all $p \in(2, \infty)$. However, (2.3), Hölder's inequality, the Sobolev embedding theorem and the fact $I_{\lambda_{n}}^{\prime}\left(u_{\lambda_{n}}\right) u_{\lambda_{n}}^{+}=0$ imply that

$$
\begin{aligned}
\left\|u_{\lambda_{n}}^{+}\right\|^{2} & =\lambda_{n} \int_{\mathbb{R}}\left(\nabla W\left(t, u_{\lambda_{n}}\right), u_{\lambda_{n}}^{+}\right) d t \\
& \leq \varepsilon \int_{\mathbb{R}}\left|u_{\lambda_{n}}\right| \cdot\left|u_{\lambda_{n}}^{+}\right| d t+C_{\varepsilon} \int_{\mathbb{R}}\left|u_{\lambda_{n}}\right|^{p-1}\left|u_{\lambda_{n}}^{+}\right| d t \\
& \leq \varepsilon\left\|u_{\lambda_{n}}\right\| \cdot\left\|u_{\lambda_{n}}^{+}\right\|+C_{\varepsilon}^{\prime}\left\|u_{\lambda_{n}}\right\|_{L^{p}}^{p-1}\left\|u_{\lambda_{n}}^{+}\right\| \\
& \leq \varepsilon\left\|u_{\lambda_{n}}\right\| \cdot\left\|u_{\lambda_{n}}^{+}\right\|+C_{\varepsilon}^{\prime \prime}\left\|u_{\lambda_{n}}\right\|_{L^{p}}^{p-2}\left\|u_{\lambda_{n}}\right\| \cdot\left\|u_{\lambda_{n}}^{+}\right\| \\
& \leq \varepsilon\left\|u_{\lambda_{n}}\right\|^{2}+C_{\varepsilon}^{\prime \prime}\left\|u_{\lambda_{n}}\right\|_{L^{p}}^{p-2}\left\|u_{\lambda_{n}}\right\|^{2} .
\end{aligned}
$$

Similarly, we have

$$
\left\|u_{\lambda_{n}}^{-}\right\|^{2} \leq \varepsilon\left\|u_{\lambda_{n}}\right\|^{2}+C_{\varepsilon}^{\prime \prime}\left\|u_{\lambda_{n}}\right\|_{L^{p}}^{p-2}\left\|u_{\lambda_{n}}\right\|^{2}
$$

Therefore,

$$
\left\|u_{\lambda_{n}}\right\|^{2} \leq 2 \varepsilon\left\|u_{\lambda_{n}}\right\|^{2}+2 C_{\varepsilon}^{\prime \prime}\left\|u_{\lambda_{n}}\right\|_{L^{p}}^{p-2}\left\|u_{\lambda_{n}}\right\|^{2}
$$

which means $\left\|u_{\lambda_{n}}\right\|_{L^{p}} \geq C$ for some constant $C$. Hence $\left\{u_{\lambda_{n}}\right\}$ is non-vanishing. Let us define $v_{\lambda_{n}}=u_{\lambda_{n}}\left(\cdot-s_{n}\right)$, from $\lim _{n \rightarrow \infty} \int_{B_{r}\left(s_{n}\right)}\left|u_{\lambda_{n}}\right|^{2} d t \geq \delta$, we have

$$
\lim _{n \rightarrow \infty} \int_{B_{r}(0)}\left|v_{\lambda_{n}}\right|^{2} d t \geq \frac{\delta}{2}
$$

Note that $I$ and $I^{\prime}$ are both invariant under translation, so we know $I^{\prime}\left(v_{\lambda_{n}}\right) \rightarrow 0$. Since $\left\{v_{\lambda_{n}}\right\}$ is still bounded, we may assume $v_{\lambda_{n}} \rightarrow u$ in E. By Lemma 2.2, $v_{\lambda_{n}} \rightarrow u$ in $L_{\text {loc }}^{2}\left(\mathbb{R}, \mathbb{R}^{2 N}\right)$. It follows from (3.1) that $u \neq 0$ with $I^{\prime}(u)=0$.

Let $K:=\left\{u \in E \mid I^{\prime}(u)=0, u \neq 0\right\}$ be the critical set of $I$ and $c:=$ $\inf \{I(z) \mid z \in K \backslash\{0\}\}$. For any critical point $u$ of $I$, assumption $\left(\mathrm{W}_{2}\right)$ implies that

$$
I(u)=I(u)-\frac{1}{2} I^{\prime}(u) u=\int_{\mathbb{R}}\left(\frac{1}{2}(\nabla W(t, u), u)-W(t, u)\right) d t>0 \quad \text { if } u \neq 0 .
$$

Therefore, we have $c \geq 0$. We prove that $c>0$ and there is $u \in K$ such that $I(u)=c$. Let $u_{j} \in K \backslash\{0\}$ be such that $I\left(u_{j}\right) \rightarrow c$. Then, the proof of Lemma 2.5 
shows that $\left\{u_{j}\right\}$ is bounded, then by the concentration compactness principle discussion above we know $u_{j} \rightarrow u \in K \backslash\{0\}$. Thus

$$
\begin{aligned}
c=\lim _{j \rightarrow \infty} I\left(u_{j}\right) & =\lim _{j \rightarrow \infty}\left(I\left(u_{j}\right)-\frac{1}{2} I^{\prime}\left(u_{j}\right) u_{j}\right) \\
& =\lim _{j \rightarrow \infty} \int_{\mathbb{R}}\left(\frac{1}{2}\left(\nabla W\left(t, u_{j}\right), u_{j}\right)-W\left(t, u_{j}\right)\right) d t \\
& \geq \int_{\mathbb{R}}\left(\frac{1}{2}(\nabla W(t, u), u)-W(t, u)\right) d t=I(u) \geq c,
\end{aligned}
$$

where the first inequality is due to Fatou's lemma. So $I(u)=c$ and $c>0$ because $u \neq 0$.

Proof of Theorem 1.4. By virtue of Theorem 1.3, it suffices to show that (1.1) has no nontrivial homoclinic orbit if $\left(\mathrm{A}_{1}\right),\left(\mathrm{W}_{1}\right)-\left(\mathrm{W}_{4}\right)$ with $V(t) \equiv V$, $|\nabla W(t, u)| /|u| \leq V$ for all $(t, u) \in \mathbb{R} \times \mathbb{R}^{2 N}$ and $V \leq \Lambda_{0}:=\min \{-\underline{\Lambda}, \bar{\Lambda}\}$ hold. By way of contradiction, we assume that (1.1) has a nontrivial homoclinic orbit $u \in E$, then for any $\varepsilon>0$ there exists $R>0$ such that $|u(t)|<\varepsilon$ if $|t| \geq R$. It follows from (2.1), (2.2), ( $\left.\mathrm{W}_{1}\right), I^{\prime}(u)\left(u^{+}-u^{-}\right)=0,|\nabla W(t, u)| /|u| \leq V$ for all $(t, u) \in \mathbb{R} \times \mathbb{R}^{2 N}$ and $V \leq \Lambda_{0}:=\min \{-\underline{\Lambda}, \bar{\Lambda}\}$ that

$$
\begin{aligned}
&\left(B u^{+}, u^{+}\right)_{L^{2}}-\left(B u^{-}, u^{-}\right)_{L^{2}}=\left(B u, u^{+}-u^{-}\right)_{L^{2}}=\int_{\mathbb{R}}\left(\nabla W(t, u), u^{+}-u^{-}\right) d t \\
& \leq \int_{\{t \in \mathbb{R} \| t \mid \leq R\}} \frac{|\nabla W(t, u)|}{|u|}|u| \cdot\left|u^{+}-u^{-}\right| d t \\
&+\int_{\{t \in \mathbb{R}|| t \mid \geq R\}} \frac{|\nabla W(t, u)|}{|u|}|u| \cdot\left|u^{+}-u^{-}\right| d t \\
&< \int_{\mathbb{R}} V|u| \cdot\left|u^{+}-u^{-}\right| d t \leq\left(\int_{\mathbb{R}} V u^{2} d t\right)^{1 / 2}\left(\int_{\mathbb{R}} V\left(u^{+}-u^{-}\right)^{2} d t\right)^{1 / 2} \\
& \leq \Lambda_{0}\left(\int_{\mathbb{R}} u^{2} d t\right)^{1 / 2}\left(\int_{\mathbb{R}}\left(u^{+}-u^{-}\right)^{2} d t\right)^{1 / 2} \\
&= \Lambda_{0}\left\|u^{+}\right\|_{L^{2}}^{2}+\Lambda_{0}\left\|u^{-}\right\|_{L^{2}}^{2} \leq \bar{\Lambda}\left\|u^{+}\right\|_{L^{2}}^{2}-\underline{\Lambda}\left\|u^{-}\right\|_{L^{2}}^{2} .
\end{aligned}
$$

That is, $\left(B u^{+}, u^{+}\right)_{L^{2}}-\left(B u^{-}, u^{-}\right)_{L^{2}}<\bar{\Lambda}\left\|u^{+}\right\|_{L^{2}}^{2}-\underline{\Lambda}\left\|u^{-}\right\|_{L^{2}}^{2}$. However, by $\left(\mathrm{A}_{1}\right)$, we have

$$
\left(B u^{+}, u^{+}\right)_{L^{2}}-\left(B u^{-}, u^{-}\right)_{L^{2}} \geq \bar{\Lambda}\left\|u^{+}\right\|_{L^{2}}^{2}-\underline{\Lambda}\left\|u^{-}\right\|_{L^{2}}^{2} .
$$

Therefore, we get a contradiction, which completes the proof.

\section{REFERENCES}

[1] G. Arioli And A. Szulkin, Homoclinic solutions of Hamiltonian systems with symmetry, J. Differential Equations, 158 (1999), 291-313.

[2] G. Chen And S. MA, Homoclinic orbits of superlinear Hamiltonian systems, Proc. Amer. Math. Soc. 139 (2011), 3973-3983. 
[3] V. Coti-Zelati, I. Ekeland and E. SÉRÉ, A variational approach to homoclinic orbits in Hamiltonian systems, Math. Ann. 288 (1990), 133-160.

[4] Y.H. DiNG, Multiple homoclinics in a Hamiltonian system with asymptotically or super linear terms, Commun. Contemp. Math. 8 (2006), 453-480.

[5] _ Variational Methods for Strongly Indefinite Problems, Interdiscip. Math. Sci., vol. 7, World Scientific, 2007.

[6] Y.H. Ding and M. Girard, Infinitely many homoclinic orbits of a Hamiltonian system with symmetry, Nonlinear Anal. 38 (1999), 391-415.

[7] Y.H. Ding And M. Willem, Homoclinic orbits of a Hamiltonian system, Z. Angew. Math. Phys. 50 (1999), 759-778.

[8] H. Hofer And K. Wysocki, First order elliptic systems and the existence of homoclinic orbits in Hamiltonian systems, Math. Ann., 228 (1990), 483-503.

[9] P.L. Lions, The concentration-compactness principle in the calculus of variations: The locally compact cases, Parts I and II, Ann. Inst. H. Poincaré Anal. Non Linéaire 1 (1984), 223-283.

[10] E. SÉRÉ, Existence of infinitely many homoclinic orbits in Hamiltonian systems, Math. Z. 209 (1992), 27-42.

[11] L Looking for the Bernoulli shift, Ann. Inst. H. Poincaré Anal. Non Linéaire, 10 (1993), 561-590.

[12] M. Schechter And W. Zou, Weak linking theorems and Schrödinger equations with critical Sobolev exponent, ESAIM Control Optim. Calc. Var. 9 (2003), 601-619.

[13] K. TANAKa, Homoclinic orbits in a first order super-quadratic Hamiltonian system: convergence of subharmonic orbits, J. Differential Equations 94 (1991), 315-339.

[14] M. Willem, Minimax Theorems, Birkhäuser, 1996.

[15] X. XU, Homoclinic orbits for first order hamiltonian systems possessing super-quadratic potentials, Nonlinear Anal. 51 (2002), 197-214.

[16] S. ZhANG, Symmetrically Homoclinic Orbits for Symmetric Hamiltonian Systems, J. Math. Anal. Appl. 247 (2000), 645-652.

Manuscript received September 10, 2014

accepted December 14, 2014

Guanwei Chen

School of Mathematical Sciences

University of Jinan

Jinan 250022, Shandong Province, P.R. CHINA

E-mail address: guanweic@163.com 